\title{
Neuropsychological and psychiatric differences between Alzheimer's disease and Parkinson's disease with dementia
}

\author{
Sergio E Starkstein, Liliana Sabe, Gustavo Petracca, Erán Chemerinski, Gabriela Kuzis, \\ Marcelo Merello, Ramón Leiguarda
}

\begin{abstract}
Objective-To examine neuropsychological and neuropsychiatric differences between patients with probable Alzheimer's disease and patients with Parkinson's disease and dementia.

Methods-Thirty three patients with probable Alzheimer's disease and 33 patients with Parkinson's disease and dementia were matched for age, sex, and mini mental state examination scores and given a battery of neuropsychological and neuropsychiatric tests.

Results-Patients with Parkinson's disease with dementia had a significantly higher prevalence of major depression than patients with Alzheimer's disease; patients with Alzheimer's disease showed more severe anosognosia and disinhibition than patients with Parkinson's disease. Whereas no significant between group differences were found on tests of memory and language, demented patients with Parkinson's disease had a significantly greater impairment on a test of visual reasoning than patients with Alzheimer's disease.

Conclusion-There were significant psychiatric differences between patients with Alzheimer's disease and demented patients with Parkinson's disease, but neuropsychological differences were restricted to a single cognitive domain.
\end{abstract}

$(\mathcal{F}$ Neurol Neurosurg Psychiatry 1996;61:381-387)

Department of Behavioral Neurology

S E Starkstein

L Sabe

G Petracca

E Chemerinski

G Kuzis

Department of Clinical

Neurology, Raúl

Carrea Institute of

Neurological Research,

Buenos Aires,

Argentina

S E Starkstein

M Merello

R Leiguarda

Correspondence to:

Dr Sergio E Starkstein, Raúl

Carrea Institute of

Neurological Research,

Montañeses 2325, 1428

Buenos Aires, Argentina.

Received 18 March 1996

and in revised form

28 May 1996

Accepted 6 June 1996 disease

Alzheimer's disease and dementia in tical dementias, whereas apathy or depression may be more prevalent in subcortical dementias. $^{1}$ Finally, they also pointed out that although motor abnormalities may be absent in cortical dementias, disorders of movement, tone, posture, and gait are universal in subcortical dementias.

None the less, the classification of the dementias into cortical and subcortical types is a controversial issue. Several authors suggested that this classification scheme should be abandoned, ${ }^{23}$ because at necropsy, brains of patients with cortical dementias also feature subcortical pathological change (for example, depletion of cholinergic neurons in the nucleus basalis of Meynert in Alzheimer's disease), and similarly, brains of patients with subcortical dementias may also show cortical damage (for example, cortical atrophy in Huntington's disease). ${ }^{1}$ Another confounding issue is that cortical and subcortical dementias may coexist in the same patient. For example, several studies reported patients with Parkinson's disease and dementia who also showed the characteristic neuropathological features of Alzheimer's disease. ${ }^{4}$

Neuropsychological differences between patients with Alzheimer's disease and demented patients with Parkinson's disease have been examined in several studies. Whereas Mayeux et al could not find significant differences between patients with Alzheimer's disease and demented patients with Parkinson's disease on a test of global cognitive functions, ${ }^{5}$ studies using more detailed neuropsychological assessments showed significant between group differences. ${ }^{6}$

Keywords: dementia; Alzheimer's disease; Parkinson's ${ }^{7}$ Affective changes have also been reported in both Alzheimer's disease and Parkinson's disease. Most studies showed either dysthymia or major depression in about one third of patients with Parkinson's disease, ${ }^{8}$ and a similar prevalence of depression has been reported in Alzheimer's disease. ${ }^{9}$ However, the prevalence of depression among patients with Parkinson's disease and dementia has not been examined, and to our knowledge, whether affective disorders have a different prevalence in Alzheimer's disease and patients with Parkinson's disease and dementia has not been assessed. Moreover, whereas Cummings and Benson ${ }^{1}$ speculated that patients with Alzheimer's disease may be more anosognosic than demented patients with Parkinson's disease, this hypothesis has not been empirically examined.

Motor disorders such as tremor, rigidity, and akinesia are the hallmark of Parkinson's disease, but they are also present in about a 
quarter of patients with Alzheimer's disease. ${ }^{10}$ In a recent study, we showed significant cognitive and psychiatric differences between patients with Alzheimer's disease with or without extrapyramidal motor signs. ${ }^{11}$ Thus to compare cognitive and psychiatric functioning in patients with Alzheimer's disease and patients with Parkinson's disease and dementia it is important to screen patients with Alzheimer's disease for the presence of extrapyramidal signs.

Finally, to our knowledge studies using the same comprehensive neuropsychological, neuropsychiatric, and neurological assessments in cohorts of Alzheimer's disease and demented patients with Parkinson's disease have not been carried out. This was the aim of the present study. Patients with Alzheimer's disease and patients with Parkinson's disease and dementia were matched for age, sex, and global cognitive performance. None of the patients with Alzheimer's disease had resting tremor, cogwheel rigidity, or bradykinesia. These groups were compared for cognitive performance and psychiatric symptoms.

\section{Patients and methods}

PATIENTS

A consecutive series of patients with either probable Alzheimer's disease or Parkinson's disease who attended the neurology clinic of the Raúl Carrea Institute of Neurological Research were screened for participation in the study.

\section{Alzheimer's disease group}

Patients with Alzheimer's disease were selected from a group of 204 consecutive patients followed up in the neurology clinic of our Institute. Patients included in this group met the NINCDS-ADRDA criteria for probable Alzheimer's disease. ${ }^{11}$ All patients were examined with the unified Parkinson's disease rating scale, ${ }^{12}$ and only patients rating 0 on items of rigidity, tremor, and bradykinesia were included.

Group with dementia and Parkinson's disease This group comprised 33 consecutive patients meeting both the United Kingdom Parkinson's Disease Society brain bank clinical criteria for Parkinson's disease ${ }^{13}$ and the DSM-IV criteria of dementia due to Parkinson's disease ${ }^{14}$ who were examined between January 1993 and December 1994. All patients had typical clinical features of Parkinson's disease and responded to levodopa. Patients with a history of cognitive decline before or less than one year after the onset of Parkinson's disease were excluded. Thirty three out of a consecutive series of 111 patients with Parkinson's disease were included in the study. All the demented patients with Parkinson's disease were assessed while in the "on" state.

Each of the 33 demented patients with Parkinson's disease were matched for age $( \pm 2$ years), sex, and mini mental state examination score ( \pm 1 point) with one patient with probable Alzheimer's disease.

\section{NEUROLOGICAL EXAMINATION}

After informed consent, patients were assessed by a neurologist with the Unified Parkinson's disease rating scale (UPDRS), ${ }^{12}$ a widely used scale for assessing the severity of clinical symptoms in patients with Parkinson's disease. This scale has three sections: activities of daily living, motor examination, and complications of antiparkinsonian treatment. Item scores range from 0 to 4 , and higher scores indicate more severe impairments.

\section{PSYCHIATRIC EXAMINATION}

The psychiatric evaluation was completed by a psychiatrist blind to the UPDRS scores and neuropsychological findings, and included the following assessments:

\section{Structured clinical interview for DSM-III-R (SCID) ${ }^{15}$}

The SCID is a semistructured diagnostic interview assessing signs and symptoms necessary for the major axis I DSM-III-R diagnoses. The interview was carried out with the patient and at least one first degree relative. Based on the SCID responses, DSM-III-R diagnoses of major depression and dysthymia were made.

\section{Hamilton depression scale $(H A M-D)^{16}$}

The HAM-D is a 17 item interviewer rated scale for rating the severity of symptoms of depression.

\section{Anosognosia questionnaire-dementia $(A Q-D)^{17}$}

This instrument consists of 30 questions divided into two sections. The first section assesses intellectual functioning (for example, Do you have problems remembering dates? Do you have problems remembering telephone calls?), and the second section examines changes in interests and personality (for example, Do you get easily irritated? Have you lost interest in things?). Each answer is rated as never ( 0 points), sometimes ( 1 point), usually ( 2 points), and always present ( 3 points). Thus higher scores indicate more severe impairments. Form $\mathrm{A}$ is answered by the patient alone, and form B (a similar questionnaire written in the third person) is answered by the patient's care giver blind to the patient's answers in form A. The final score is the difference between total scores obtained by substracting form $B$ from form $A$. Thus positive scores indicate that the care giver rated the patient as more impaired than the patient's own evaluation (the patient was less aware of his or her cognitive and emotional deficits). We have recently showed the reliability and validity of this scale.

\section{Bech mania scale ${ }^{18}$}

This scale assesses the presence and severity of manic symptoms, such as euphoria, hyperactivity, flight of ideas, irritability, hypersexuality, and grandiose delusions.

\section{Pathological laughing and crying scale (PLACS) ${ }^{19}$}

This instrument is an interviewer rated scale that quantifies aspects of pathological affect, 
including the duration of the episodes, their relation to external events, degree of voluntary control, inappropriateness in relation to emotions, and degree of resultant distress. The reliability and validity of this scale in patients with Alzheimer's disease have been previously established. The scale is administered to the patient and at least one first degree relative or care giver in close contact with the patient. The scale consists of 16 items (eight assessing pathological laughter (PLACS-L) and eight assessing pathological crying (PLACS-C)), which are scored from 0 to 3 points.

Apathy scale ${ }^{20}$

The apathy scale includes 14 items which are scored by the patient's relative or care giver. Each question has four possible answers, which are scored from 0 to 3 . Thus the apathy scale score ranges from 0 to 42 points, and higher scores indicate more severe apathy. We have verified the reliability and validity of the apathy scale in Alzheimer's disease. ${ }^{21}$

\section{Irritability scale ${ }^{21}$}

This is a 14 item scale which is rated by the patient's relative or a care giver. Scores range from 0 to 42 , and higher scores indicate more severe irritability. We have verified the validity and reliability of this scale in Alzheimer's disease. $^{21}$

\section{Dementia psychosis scale ${ }^{22}$}

This 18 item scale quantifies the severity and types of delusions in demented patients at the time of the psychiatric evaluation. It is completed by a psychiatrist during an interview with the patient and at least one close relative or care giver. We have shown this scale to be valid and reliable in Alzheimer's disease. ${ }^{22}$

Functional independence measure $e^{23}$

This instrument assesses self care, sphincter control, mobility, locomotion, communication, and social cognition on a low level scale. Higher scores indicate less impairments in activities of daily living.

\section{Social ties checklist ${ }^{24}$}

This is a 10 item scale which assesses the quantity and quality of social supports. Scores range from 0 to 10 , and higher scores indicate better social supports.

\section{NEUROPSYCHOLOGICAL EXAMINATION}

Each patient was assessed by a neuropsychologist blind to neurological and psychiatric findings with the following test battery:

\section{Mini mental state exam (MMSE) ${ }^{25}$}

MMSE is an 11 item examination that has been found to be reliable and valid in assessing general cognitive functions and serves as a cognitive screening instrument in patients with dementia.

\section{Buschke selective reminding test ${ }^{26}$}

This test measures verbal learning and memory during a multiple trial list learning task. The patient listens to a list of words and recalls as many words as possible. Each subsequent learning trial involves the presentation of only those words that were not recalled on the immediately preceding trial. The outcome measure was the number of words in long term storage.

\section{Benton visual retention test ${ }^{27}$}

This test assesses visual perception and nonverbal memory. Patients are exposed to geometric designs for 10 seconds, and are immediately presented with a card containing the correct design among three foils. The patient is asked to select the previously presented design. There are 10 trials.

\section{Digit span ${ }^{28}$}

This subtest of the Wechsler memory scale examines auditory attention, and includes two parts. Both consist of seven pairs of number sequences that the examiner presents at the rate of one per second. In the first part (digits forward), the patient is asked to simply repeat a string of numbers (from two to eight numbers in length) exactly as it is given. In the second part (digits backwards) the patient is asked to repeat the string of numbers (from two to eight numbers in length) in reversed order.

Wisconsin card sorting test (WCST) ${ }^{29}$

This test measures the ability to develop and apply new concepts, and, subsequently shift sets, which requires the subjects to suppress a learned response that was previously correct and learn a new one. Assessment of the overall proficiency of the test was judged by the number of categories achieved (maximum 6).

\section{Controlled oral word association test ${ }^{30}$}

This test examines access to semantic information with time constraint. Patients were instructed to name as many words beginning with the letter $\mathrm{F}$ as they could in one minute. People's names and proper nouns were not permitted. The letters $A$ and $S$ were then presented successively, one minute being allowed for each letter. The score was the combined number of appropriate words produced in three minutes.

\section{Raven's progressive matrices ${ }^{31}$}

This test measures visuospatial reasoning. Patients are presented with a spatial pattern problem with one part removed and four pictured inserts, one of which contains the correct pattern. The patient has to select the correct piece to match the original spatial patterns. The patterns become increasingly complex over trials. The performance score is the number correctly identified.

\section{Purdue pegboard test ${ }^{32}$}

This test assesses manipulative dexterity. The apparatus consists of a board containing two parallel rows of 25 holes each and 50 metal pegs. Patients are asked to take the pegs with the preferred (for example, right) hand and place them as quickly as possible in the right column of holes during a 30 second period. The same procedure is repeated with the non- 
Table 1 Demographic and neurological findings

\begin{tabular}{lll}
\hline & $P D$ dementia group & AD group \\
\hline No of patients & 33 & 33 \\
Age (mean y) & $71 \cdot 0(8 \cdot 5)$ & $70 \cdot 3(8 \cdot 2)$ \\
Sex (\% women) & 30 & 30 \\
Education (mean y) & $9 \cdot 6(5 \cdot 9)$ & $10 \cdot 5(5 \cdot 7)$ \\
UPDRS motor score (mean scores) & $22 \cdot 9(9 \cdot 8)$ & $0 \cdot 6(1 \cdot 1)$ \\
\hline
\end{tabular}

Values in parentheses are SD. $\mathrm{PD}=$ Parkinson's disease; $\mathrm{AD}=$ Alzheimer's disease

$\star \star \star \mathrm{P}<0.0001$.

preferred hand, and the score is the number of pins inserted in the time for each hand.

\section{STATISTICAL ANALYSIS}

Groups were compared by multivariate analysis of covariance (MANCOVA), and post hoc tests, when appropriate. Frequency distributions of dichotomous variables were examined with contingency tables, and $\chi^{2}$ tests were calculated with a Yates' correction for cell sizes $<5$. For all analyses, tests were two tailed.

\section{Results}

DEMOGRAPHIC AND NEUROLOGICAL FINDINGS (TABLE 1)

The Alzheimer's disease and the demented Parkinson's disease groups were matched for age and sex, and there were no significant between group differences in education. One patient with Parkinson's disease (3\%) was in Hoehn and Yahr stage 1, 10 patients (31\%) were in stage 2,16 patients $(48 \%)$ in stage 3 , five patients $(15 \%)$ in stage 4 , and one $(3 \%)$ in stage 5 . The mean (SD) daily levodopa dosage for the Parkinson's disease-dementia group was 519 (410) (range: 0-1250) $\mathrm{mg} /$ day, and none of the patients were on anticholinergic drugs.

PSYCHIATRIC FINDINGS (TABLE 2)

Demented patients with Parkinson's disease showed significantly higher HAM-D scores

Table 2 Psychiatric findings

\begin{tabular}{llc}
\hline & PD dementia group & AD group \\
\hline Hamilton depression scale $_{\text {Major depression (\% patients) }}^{\star}$ & $12 \cdot 6(8 \cdot 8)$ & $7 \cdot 1(5 \cdot 6)$ \\
Dysthymia (\%) & 30 & 6 \\
No depression (\%) & 27 & 27 \\
Bech mania scale (mean) & 43 & 67 \\
Psychosis dementia scale (mean) & 0 & $2 \cdot 0(5 \cdot 9)$ \\
Functional independence measure & $1 \cdot 0(1 \cdot 9)$ & $1 \cdot 7(2 \cdot 7)$ \\
Anosognosia quest-dementia & $60 \cdot 9(12 \cdot 4)$ & $64 \cdot 7(8 \cdot 0)$ \\
Irritability scale & $2 \cdot 2(14 \cdot 1)$ & $12 \cdot 1(22 \cdot 4)$ \\
Apathy scale & $10 \cdot 4(7 \cdot 3)$ & $12 \cdot 6(9 \cdot 3)$ \\
Pathological laughing scale & $17 \cdot 8(7 \cdot 9)$ & $15 \cdot 5(11 \cdot 1)$ \\
Pathological crying scale & 0 & $0 \cdot 5(2 \cdot 6)$ \\
Social ties checklist & $2 \cdot 1(3 \cdot 7)$ & $4 \cdot 4(5 \cdot 3)$ \\
\hline
\end{tabular}

Values in parentheses are SD.

$\star P<0.05 ;+P<0.05$.

Table 3 Neuropsychological findings

\begin{tabular}{lcc}
\hline & PD dementia group & AD group \\
\hline Mini mental state examination & $20 \cdot 3(6 \cdot 5)$ & $20 \cdot 3(6 \cdot 2)$ \\
Raven's progressive matrices (\%) $^{\star}$ & $24 \cdot 7(23 \cdot 2)$ & $48 \cdot 3(34 \cdot 1)$ \\
Winconsin card sorting test (categories) & $2 \cdot 6(2 \cdot 1)$ & $2 \cdot 5(2 \cdot 0)$ \\
Word controlled association test & $28 \cdot 3(9 \cdot 2)$ & $30 \cdot 7(11 \cdot 8)$ \\
Buschke selective reminding test & $32 \cdot 3(18 \cdot 6)$ & $28 \cdot 8(23 \cdot 1)$ \\
Benton visual retention test & $5 \cdot 0(2 \cdot 2)$ & $5 \cdot 5(2 \cdot 4)$ \\
Digits forward & $5 \cdot 2(1 \cdot 3)$ & $5 \cdot 1(0 \cdot 9)$ \\
Digits backward & $3 \cdot 3(0 \cdot 9)$ & $3 \cdot 2(1 \cdot 0)$ \\
Purdue pegboard test-right $^{\star}$ & $6 \cdot 7(3 \cdot 4)$ & $9 \cdot 7(2 \cdot 2)$ \\
Purdue pegboard test-left $^{\star}$ & $6 \cdot 5(3 \cdot 3)$ & $10 \cdot 0(2 \cdot 4)$ \\
\hline
\end{tabular}

Values in parentheses are $S D$

$\star \mathrm{P}<0.05$. than patients with Alzheimer's disease ( $t=$ 2.97 , $\mathrm{df}=64, \mathrm{P}<0.01)$. Major depression was found in 10 patients $(30 \%)$ with Parkinson's disease and dementia and two patients (6\%) with Alzheimer's disease, and dysthymia was found in nine patients $(27 \%)$ with Parkinson's disease and dementia and nine patients $(27 \%)$ with Alzheimer's disease. A hypothesis of unequal frequency of major depression based on the presence of Parkinson's disease-dementia or Alzheimer's disease was statistically substantiated $\left(\chi^{2}=\right.$ $7 \cdot 11$, df $=2, \mathrm{P}<0.05)$. On the other hand, patients with Alzheimer's disease showed significantly higher $A Q-D$ scores (more severe anosognosia; $t=2.0, \mathrm{df}=64, \mathrm{P}<0.05)$ and significantly higher mania scores than demented patients with Parkinson's disease $(t=1.96, \mathrm{df}=64, \mathrm{P}=0.05)$. No significant between group differences were found for the remaining psychiatric variables.

\section{NEUROPSYCHOLOGICAL FINDINGS (TABLE 3)}

Due to scheduling problems or patients' refusal three patients with Alzheimer's disease and five with dementia and Parkinson's disease could only complete part of the neuropsychological evaluation and had to be excluded from the statistical analysis. However, no significant differences in age and MMSE scores were found between the groups with Alzheimer's disease or Parkinson's disease with dementia that completed the neuropsychological examination (age: Alzheimer's disease group (mean (SD) 69.9 (8.3), Parkinson's disease-dementia group 70.5 $(8 \cdot 1)(F(1,56)=0 \cdot 07, \mathrm{NS})$; MMSE scores $21 \cdot 1(5 \cdot 7)$ v $20 \cdot 0(6 \cdot 7)$ respectively $(\mathrm{F}(1,56)$ $=0.44$, NS)). MANCOVA for neuropsychological tasks (using HAM-D scores as a covariate) showed a significant main effect (Wilks' lambda $=0.70, \mathrm{df}=7,45, \mathrm{P}<0.05$ ). On individual comparisons, patients with Parkinson's disease and dementia had a significantly worse performance on the Raven's progressive matrices $(\mathrm{F}(1,51)=8 \cdot 10, \mathrm{P}<0.01)$. The Parkinson's disease with dementia group also showed a significantly worse performance on the Purdue pegboard test than patients with Alzheimer's disease (right hand $F(1,53)=15.4, P<0.001)$; left hand $F(1,53)$ $=22.7, P<0.0001)$. No other significant between group differences were found for the remaining neuropsychological tasks.

\section{Discussion}

We compared the type and severity of cognitive impairments as well as the prevalence of psychiatric disorders in groups of patients with either Alzheimer's disease or Parkinson's disease and dementia. The groups were matched for age, sex, and MMSE score. Significant between group differences in both neuropsychological and psychiatric variables were noted. Demented patients with Parkinson's disease had significantly more severe deficits on a task of visuospatial reasoning and performed more slowly on a motor task than did patients with Alzheimer's disease. Demented 
patients with Parkinson's disease showed a significantly higher frequency of major depression but patients with Alzheimer's disease showed more severe anosognosia and disinhibition.

Some limitations of our study should be pointed out. One important issue is whether patients with Parkinson's disease and dementia may have had other psychiatric diagnoses, such as Alzheimer's disease with extrapyramidal signs. This is unlikely as all our demented patients with Parkinson's disease had a positive response to levodopa, and we have recently shown that patients with Alzheimer's disease with extrapyramidal signs do not have improved motor function after levodopa. ${ }^{10}$ Moreover, all our patients with Parkinson's disease and dementia had the onset of cognitive impairment more than one year after the onset of motor deficits. The second limitation was that we had no neuropathological confirmation of our clinical diagnoses, and whether our patients with Parkinson's disease may have the neuropathological changes of a diffuse Lewy body disease cannot be ruled out. However, whereas patients with diffuse Lewy body disease usually show no or only a mild and transient response to dopaminergic agonists, ${ }^{33}$ our patients with Parkinson's disease and dementia showed a consistently positive response to levodopa. McKeith et al have recently proposed that the presence of fluctuation cognitive impairments affecting both memory and higher cortical functions, as well as hallucinations and paranoid delusions, mild spontaneous extrapyramidal features, and repeated unexplained falls may characterise patients with Lewy body dementia. ${ }^{34}$ As this neuropsychiatric syndrome usually starts before the onset of extrapyramidal signs, it is highly unlikely that patients with Lewy body dementia were included in our group with Parkinson's disease and dementia. Another limitation is that we assessed our patients with Parkinson's disease after the intake of their regular dose of levodopa. Although their cognitive performance might have been worse without levodopa, several studies could not show significant differences in cognitive performance for on versus off levodopa among patients with Parkinson's disease. ${ }^{35}$ The possible influence of levodopa on behavioural problems such as apathy and anosognosia should be examined in future studies. Finally, it could be argued that among patients with Parkinson's disease the HAM-D may rate extrapyramidal symptoms as depressive complaints. However, we showed the validity of the autonomic and affective symptoms of depression rated in the HAM-D for the diagnosis of dysthymia and major depression in Parkinson's disease. ${ }^{36}$

Cummings and Benson ${ }^{1}$ suggested that cortical and subcortical dementias differ in terms of motor disorders, profile of cognitive deficits, and prevalence of psychiatric disorders, but to our knowledge no systematic study of all these variables in the same cohort of patients has been carried out. Our study offers some support for the hypothesis of Cummings and
Benson: the demented group with Parkinson's disease showed more severe motor slowing and a higher prevalence of major depression, whereas patients with Alzheimer's disease showed significantly more severe anosognosia and disinhibition. On the other hand, between group differences on neuropsychological testing were restricted to a single task of visuospatial reasoning.

Several studies have examined neuropsychological differences between patients with cortical and subcortical dementias (mostly Alzheimer's disease $v$ Parkinson's disease, Huntington's disease, or progressive supranuclear palsy). ${ }^{6737-39}$ Some studies using screening measures of global cognitive performance did not find significant differences between patients with cortical and subcortical dementias, ${ }^{5}$ but studies using more comprehensive neuropsychological evaluations have shown significant between group differences. ${ }^{6} 738-40$ Cummings et $a^{39}$ compared patients with Alzheimer's disease and demented patients with Parkinson's disease and a similar global severity of cognitive impairment. They found that whereas patients with Alzheimer's disease had more severe anomia and less information content in spontaneous speech, demented patients with Parkinson's disease had more grammatically simplified utterances and dysarthria. Freedman and Oscar-Berman ${ }^{40}$ reported greater impairment on a delayed alternation task in patients with Alzheimer's disease than in demented patients with Parkinson's disease, but no significant between group differences on delayed response tasks. Litvan et $a l^{8}$ reported more severe deficits on tests of executive function among demented patients with Parkinson's disease, whereas patients with Alzheimer's disease showed greater impairment on tasks of semantic and episodic memory. Similar findings were reported by Pillon et al. ${ }^{6}$

In the present study we found demented patients with Parkinson's disease to be significantly more impaired on the Raven's progressive matrices than patients with Alzheimer's disease with a similar severity of dementia. This test requires the subject to conceptualise spatial, design and numerical relations, ranging from very concrete to very abstract designs. Deficits in visuospatial perception and abstraction have been reported in patients with Parkinson's disease even in the absence of overt dementia, ${ }^{41}$ and it is possible that deficits in visuospatial reasoning are further compromised early in the dementia of Parkinson's disease. Similar findings were reported by Sahakian et $a l^{42}$ who found that patients with Parkinson's disease performed significantly worse than patients with Alzheimer's disease on tests of visual discrimination learning and attentional set shifting. However, we did not find significant differences between patients with Alzheimer's disease and demented patients with Parkinson's disease in other cognitive domains, such as verbal and visuospatial memory, attention, set alternation, and verbal fluency. Thus differential impairments in cognitive abilities in corti- 
cal and subcortical dementias are not widespread and may be restricted to specific cognitive domains. Most of our patients with Alzheimer's disease and demented patients with Parkinson's disease were in the early stages of the illness, which may have contributed to the small difference between groups in cognitive performance.

Whereas Cummings and Benson ${ }^{1}$ suggested that the prevalence of depression, anosognosia, and disinhibition may be different in cortical and subcortical dementias, to our knowledge ours is the first study to use a structured psychiatric evaluation to assess these symptoms in a sample of patients with Alzheimer's disease and demented patients with Parkinson's disease matched for age, sex, and severity of dementia. We found that whereas major depression was significantly more prevalent in demented patients with Parkinson's disease, anosognosia, and disinhibition were more severe in patients with Alzheimer's disease.

In a recent study that included 103 patients with Alzheimer's disease we found that 23\% had major depression and $28 \%$ had dysthymia. ${ }^{9}$ However, in the present study the prevalence of major depression in patients with Alzheimer's disease was lower. This discrepancy may be due to differences in sex distribution between the two studies. In our previous study $74 \%$ of the Alzheimer's disease sample and 22 of the $24(92 \%)$ patients with Alzheimer's disease with major depression were female patients. In the present study only $30 \%$ of the Alzheimer's disease sample and only four of the $10(40 \%)$ demented patients with Parkinson's disease with major depression were female patients $\left(\chi^{2}=10 \cdot 4, \mathrm{df}=1\right.$, $\mathbf{P}=0.001)$. Thus whereas major depression in Alzheimer's disease seems to occur significantly more often among female patients, in Parkinson's disease with dementia, major depression is more prevalent in male patients. These findings suggest that the association between major depression and either Alzheimer's disease or Parkinson's disease and dementia may be sex related, and differences in the prevalence of major depression between cortical and subcortical dementias may be related to the numbers of male and female patients in each group.

Another interesting finding was that anosognosia and disinhibition were both more severe in patients with Alzheimer's disease than in demented patients with Parkinson's disease. In a recent study we found that anosognosia in Alzheimer's disease was significantly associated with relatively lower right frontal lobe perfusion, ${ }^{43}$ whereas disinhibited behaviour was significantly associated with relatively more severe bilateral orbitofrontal and basotemporal perfusion deficits. ${ }^{44}$ Thus the higher prevalence of anosognosia and disinhibition in patients with Alzheimer's disease compared with demented patients with Parkinson's disease may be related to more severe frontotemporal cortical dysfunction.

Finally, we could not find significant between group differences in the severity of delusions, apathy, irritability, and emotional lability. This shows that, similar to neuropsychological findings, differences in psychiatric disorders between cortical and subcortical dementias are not widespread but may be restricted to specific psychiatric domains.

In conclusion, our study showed significant psychiatric differences between patients with Alzheimer's disease and patients with Parkinson's disease with dementia matched for age, sex, and severity of dementia. On the other hand, neuropsychological differences were mild and restricted to a single cognitive domain. Future studies should examine the association between sex and psychiatric disorders in demented patients, and determine whether specific deficits in cortical metabolic activity may account for psychiatric differences between cortical and subcortical dementias.

This study was partially supported by grants from the Raúl Carrea Institute of Neurological Research, the Fundacion Perez Companc, and the CONICET. We thank Dr Fred Bylsma for his most helpful comments.

1 Cummings JL, Benson DF. Dementia: a clinical approach. Boston: Butterworth-Heinemann, 1992.

2 Whitehouse PJ. The concept of cortical and subcortical dementia: another look. Ann Neurol 1986;19:1-6.

3 Cummings JL. Subcortical dementia. New York: Oxford University Press, 1990.

4 Boller F, Mizutani T, Roessman U, Gambetti P. Parkinson disease, dementia, and Alzheimer disease: clinicopathological correlations. Ann Neurol 1980;7:329-35.

5 Mayeux R, Stern Y, Rosen J, Benson DF. Is "subcortical dementia" a recognizable clinical entity? Ann Neurol

6 Pillon B, Dubois B, Lhermitte F, Agid Y. Heterogeneity of cognitive impairment in progressive supranuclear palsy, Parkinson's disease, and Alzheimer's disease. Neurology 1986;36:1179-85.

7 Paulsen JS, Butters N, Sadek JR, Johnson SA, Salmon DP, Swerdlow NR, Swenson MR. Distinct cognitive profiles of cortical and subcortical dementia in advanced illness. Neurology 1995;45:951-6.

8 Starkstein SE, Preziosi TJ, Bolduc PL, Robinson RG Depression in Parkinson's disease. $\mathcal{f}$ Nerv Ment Dis 1990;178:27-31.

9 Migliorelli R, Teson A, Sabe L, Petracchi M, Leiguarda R, Starkstein SE. Prevalence and correlates of dysthymia and major depression in Alzheimer's disease. $A m \mathcal{F}$ and major depression in

10 Merello M, Sabe L, Teson A, Migliorelli R, Petracchi M, Leiguarda R, Starkstein SE. Extrapyramidalism in Alzheimer's disease: prevalence, psychiatric, and neuAlzheimer's disease: prevalence, psychiatric, and neu-
ropsychological correlates. $\mathcal{F}$ Neurol Neurosurg Psychiatry ropsychological co

11 Mc Khann G, Drachman D, Folstein MF, Katzman R, Price D, Stadlan EM. Clinical diagnosis of Alzheimer's disease: report of the NINCDS-ADRDA Work Group under the auspices of Department of Health and Human Services Task Force on Alzheimer's Disease. Neurology 1984;34:939-44.

12 Fahn S, Elton E. UPDRS Development Committee. Unified Parkinson's disease rating scale. In: S Fahn, CD Marsden, M Goldstein, CD Calne, eds. Recent developments in Parkinson's disease. Florham Park, NJ: Macmillan, 1987,153-63.

13 Hughes AJ, Daniel SE, Kilford L, Lees AJ. Accuracy of clinical diagnosis of idiopathic Parkinson's disease: a clinico-pathological study of 100 cases. $₹$ Neurol Neurosurg ico-pathological study of
Psychiatry 1992;55:181-4.

14 American Psychiatric Press. Diagnostic and statistical manual of mental disorders, 4th ed. Washington, DC: APA, 1994

15 Spitzer RL, Williams JBW, Gibbon M, First MB. The structured clinical interview for DSM-III-R (SCID). I: History, rationale, and description. Arch Gen Psychiatry 1992;49:624-9.

16 Hamilton MA. A rating scale for depression. $f$ Neurol Neurosurg Psychiatry 1960;23:56-62.

7 Migliorelli R, Teson A, Sabe L, Petracca G, Petracchi M, Leiguarda R, Starkstein SE. Anosognosia in Alzheimer's disease: a study of associated factors. $\mathcal{f}$ Neuropsychiatry Clin Neurosci 1995;7:338-44.

18 Bech P, Kastrup M, Rafaelsen OJ. Mini-compendium of rating scales for states of anxiety, depression, mania, schizophrenia, with corresponding DSM-III syndrome. Acta Psychiatr Scand 1986;73(suppl 236):29-31.

19 Robinson RG, Parikh RM, Lipsey JR, Starkstein SE, Price TR. Pathological laughing and crying following stroke: ment study. Am f Psychiatry 1993;150:286-93. 
20 Starkstein SE, Mayberg HS, Preziosi TJ, Andrezejewski MA, Leiguarda R, Robinson RG. Reliability, validity, and clinical correlates of apathy in Parkinson's disease. $\mathcal{F}$ Neuropsychiatry Clin Neurosci 1992;4:134-9.

21 Starkstein SE, Migliorelli R, Manes F, Teson A, Petracca

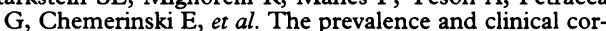
relates of apathy and irritability in Alzheimer's disease. relates of apathy and irritability in Alzheim

22 Migliorelli R, Petracca G, Teson A, Sabe L, Leiguarda R Starkstein SE. Neuropsychiatric and neuropsychologica correlates of delusions in Alzheimer's disease. Psycho Med 1995;25:505-13.

23 Granger CV, Hamilton BB, Kayton R. Guide for use of the uniform data set for medical rehabilitation. Uniform data system for medical rehabilitation. Buffalo, NY: 1986.

24 Starr LB, Robinson RG, Price TR. Reliability, validity and clinical utility of the social functioning exam in the assessment of stroke patients. Exp Aging Res 1983;9: 101-10.

25 Folstein MF, Folstein SE, McHugh PR. Mini-mental state: a practical method for grading the cognitive state of a practical method for grading the cognitive state of

26 Buschke H, Fuld PA. Evaluating storage, retention, and retrieval in disordered memory and learning. Neurology 1974;24:1019-25

27 Benton $\mathrm{AL}$. The revised visual retention test, 4 th ed. New York: Psychological Corporation, 1974

28 Wechsler D. Wechsler adult intelligence scale manual. New York: Psychological Corporation, 1955.

29 Nelson HE. A modified card sorting test sensitive to fronta lobe defects. Cortex 1976;12:313-24.

30 Benton AL. Differential behavioral effects in frontal lobe disease. Neuropsychol 1968;6:53-60.

31 Raven JC, Court JH, Raven J. Manual for Raven's progressive matrices and vocabulary scales. London: HK Lewis, 1986.

32 Tiffin J. Purdue pegboard: examiner manual. Chicago: Science Research Associates, 1968.

33 Perry RH, Irving D, Blessed G, Fairbairn A, Perry EK Senile dementia of Lewy body type: a clinically and neuropathologically distinct form of Lewy body dementia in the elderly. $\mathcal{F}$ Neurol Sci 1990;99:119-39.
34 McKeith IG, Fairbairn AF, Bothwell RA, Moore PB, Ferrier IN, Thompson P, Perry RH. An evaluation of the predictive validity and inter-rater reliability of clinical diagnostic criteria for senile dementia of Lewy body type. Neurology 1994;44:872-7.

35 Starkstein SE, Esteguy M, Berthier ML, Garcia $H$, Leiguarda R. Evoked potentials, reaction time and cognitive performance in on and off phases of Parkinson's disease. F Neurol Neurosurg Psychiatry 1989;52:338-40.

36 Starkstein SE, Preziosi TJ, Forrester AW, Robinson RG. Specificity of affective and autonomic symptoms of depression in Parkinson's disease. $\mathcal{F}$ Neurol Neurosurg Psychiatry 1990;53:869-73.

37 Brandt J, Folstein SE, Folstein MF. Differential cognitive impairment in Alzheimer's disease and Huntington's disease. Ann Neurol 1988;23:555-61.

38 Litvan Y, Mohr E, Williams J, Gomez C, Chase TN. Differential memory and executive functions in demented patients with Parkinson's and Alzheimer's disease. ₹ Neurol Neurosurg Psychiatry 1991;54:25-9.

39 Cummings JL, Darkins A, Mendez M, Hill MA, Benson DF. Alzheimer's disease and Parkinson's disease: comparison of speech and language alterations. Neurology
1988;38:680-4.

40 Freedman $M$, Oscar-Berman M. Spatial and visual learning deficits in Alzheimer's and Parkinson's disease. Brain Cogn 1989;11:1146-26.

41 Boller F, Passafiume D, Keefe NC, Rogers K, Morrow L, Kim Y. Visuospatial impairments in Parkinson's disease: role of perceptual and motor factors. Arch Neurol 1984;41:485-90.

42 Sahakian BJ, Morris RG, Evenden JL. A comparative study of visuospatial memory and learning in Alzheimer type dementia and Parkinson's disease. Brain 1988;111: 69S-718.

43 Starkstein SE, Migliorelli R, Sabe I, Teson A, Vazquez S, Leiguarda R. A SPECT study of anosognosia in Leiguarda R. A SPECT study of anosogno

44 Starkstein SE, Migliorelli R, Tesón A, Sabe L, Vázquez S, Turjanski $M$, et al. The specificity of cerebral blood flow changes in patients with frontal lobe dementia. $\mathcal{F}$ Neurol Neurosurg Psychiatry 1994;57:790-6. 